Grund mehr für Credner's Ansicht, sondern halte die drei Säuren Sulfomaleïnsiure, Sulfofumarsäure und Sulfobernsteinsäure für identisch, und kounınt denselben die Constitutionsformel :

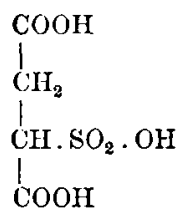

gemeinsam zu.

Für dic mir bei den vorliegenden Arbeiten von Seiten des Hern Prof. Strecker zu Theil gewordene werthvolle Hülfe spreche ich meinem hochverehrten Lehrer meinen wärmsten Dank aus.

Ueber einige aus Asparaginsäure entstehende Producte; von Dr. Eugen Schaal.

Darstellung von Asparagin aus Asparaginsüure.

Bekanntlich ist die Asparaginsäure (die optisch-unwirksame Hodification) aus den Ammoniaksalzen der Aepfelsäure, Maleinsäure oder Fumarsāure von Pasteur ${ }^{*}$ ) sowie von Dessaignes ${ }^{* *}$ ) synthetisch dargestellt worden; dagegen hat man das Asparagin bis jetzt noch nicht künstlich erhalten.

Ich habe diefs nun nach folgendem Verfahren auszuführen versucht. Zuerst war es die Aufgabe, den Monäther der

*) Diese Annalen LXXXII, 324.

**) Jasellost LXXXIII, 83. 
Asparaginsäure zu gewinnen, welcher bei der Einwirkung von Ammoniak voraussichtlich Asparagin geben würde, wie folgende Gleichung zeigt :

$$
\mathrm{C}_{2} \mathrm{H}_{8}\left\{\begin{array}{l}
\mathrm{COOH} \\
\mathrm{NH}_{8} \\
\mathrm{COOC}_{2} \mathrm{H}_{5}
\end{array}+\mathrm{NH}_{3}=\mathrm{C}_{2} \mathrm{H}_{3}\left\{\begin{array}{l}
\mathrm{COOH} \\
\mathrm{NH}_{2} \\
\mathrm{CONH}_{2}
\end{array}+\mathrm{C}_{2} \mathrm{H}_{6} \mathrm{O} .\right.\right.
$$

Reine Asparaginsäure (durch Behandeln ron Asparagin mit Barytwasser erhaiten) wurde zunächst in die Monosilberverbindung verwandelt (sic enthielt 48,4 pC. Silber, war daher mit elwas Disilberverbindung vermengt), und diese in einer Retorte mit Jodäthyl und etwas Weingeist versetzt im Wasserbade gelinde erwärınt, wobei sehr rasch die Umsetzung vor sich ging, was sich leicht an dem gebildeten Jodsilber orkennen liefs. Durch stärkeres Erwärmen wurde überschüssiges Jodälhyl mit dem Weingeist verjagt, hierauf die Masse mit Aether behandelt, wobei eine schmierige Masse zurückblieb, aus der sich aber mit Weingeist leicht der Asparaginsãureäther vollends ausziehen liefs, welcher von dem Jodsilber durch Filtration getrennt wurde.

Aus der ätherischen Lösung schieden sich bald Krystalle ab, dagegen aus der alkoholischen erst nach langem Stehen. Bei einem im Winter angestellen Versuche fanden sich auch in der alkoholischen Lösung (des optisch-unwirksamen Aethers) nach wenigen Tagen Krystalle.

Sowohl der Rückstand des ätherischen als auch der des alkoholischen Auszugs wurde mil starkem wässerigem Ammoniak verselzt; in der ersteren zeigten sich minimale Kryställchen nach einiger Zeit, dagegen in der alkoholischen selbst nach Jahresfrist nichts.

Bei einem erneuten und mit einer gröfseren Menge angestellten Versuche erhielt ich aus Asparaginsäureäther deutliche Krystalle von Asparagin in genügender Mlenge. Sie waren in ihren physikalischen Eigenschaften, sowie in 
ihrem chemischen Verhalten, mit dem natürlichen Asparagin durchaus übereinstimmend.

Auch ihre Zusammensetzung entspricht der Formel $\mathrm{C}_{4} \mathrm{II}_{3} \mathrm{~N}_{2} \mathrm{O}_{3}+\mathrm{H}_{2} \mathrm{O}$.

$0,1695 \mathrm{Grm}$. der luftrockenen Krystalle verloren bei $100^{\circ}$ C. 0,0205 Wasser, entsprechend $12,0 \mathrm{pC}$. Wasser.

$\begin{array}{ccc} & \text { Gefunden } & \text { Berechnet } \\ \text { Wasser } & 12,0 & 12,0 .\end{array}$

Bei der Stickstoffbestimmung mit Natronkalk gaben 0,147 Grm. bei $100^{\circ}$ getrockneter Substanz 0,4935 Platinsalmiak, entsprechend $21,22 \mathrm{pC}$. Stickstoff.

$\begin{array}{ccc} & \text { Gefunden } & \text { Berechnet } \\ \text { Stickstoff } & 21,22 & 21,26 .\end{array}$

Einwirlung von Salzsïure bei hoher Temperatur auf Asparagin.

Durch Einwirkung von trockener Salzsãure verlieren bekanntlich, wie A. Kohler*) und J. Preu **) gezeigt haben, sowohl Leucin als auch Alanin, welche durch blofses Kochen mit Salzsäure durchaus keine Veränderung erleiden, 1 Mol. Wasser und gehen in Leucinimid und Lactimid über. Es war zu erwarten, dafs die diesen Amidokörpern in manchen Beziehungen so ähnliche Asparaginsäure auch eine analoge Veränderung erleiden würde.

Zunächst wurde Asparagin in einem Liebig'schen Trockenapparat direct der Einwirkung trockenen Salzsäuregases ausgesetzt, und die Masse im Paraffinbad nach und nach bis zu $180^{\circ} \mathrm{C}$. erhitzt. Bei $140^{\circ}$ fing die Masse an sich stark aufzublähen und verlor viel Wasser. Nach dreitägigem Erwärmen blieb eine weifse harte trockene Masse

*) Inauguraldissertation, Tübingen 1864; diese Annal. CXXXIV, 367.

**) Inauguraldisscrtation, Tübingen 1861; diese Annal. CXXXIV, 372. 
zurück, die nun zuerst gepulvert und dann mit kaltem Wasser behandelt wurde, wobei der gröfsere Theil ungelōst blieb. Der Rückstand wurde wiederholt mit Wasser ausgekocht, bis nach sechsmaligem Kochen das Filtrat sich beim Erkalten nur noch schwach trübte. Die Hälfte des in kaltem Wasser unlöslichen Theils blieb so zurück. Beim Erkalten der kochenden Lösung schied sich eine in kaltem Wasser schwer lösliche Substanz ab.

Die Analysen der schwer löslichen Substanz ergaben Folgendes :

Bei der Verbrennung mit Kupferoxyd, chlorsaurem Kali und vorgelegten Kupferspänen gaben 0,1897 Grm. Substanz 0,313 Kohlensāure und 0,0815 Wasser. Bei der Stickstoffbestimmung mit Natronkalk gaben $0,2245 \mathrm{Grm}$. Substanz 0,4462 Platinsalmiak (oder 0,191 Platin). Diefs ist in Procenten :

$$
\begin{array}{r}
45,0 \text { Kohlenstoff } \\
4,8 \text { Wasserstoff } \\
12,4 \text { Stickstoff. }
\end{array}
$$

Auf die bisher angeführte Weise konnten nur wenige Gramme Asparagin in Arbeit genommen werden, und auch diefs war schwierig, weil die sich aufblähende Masse wiederholt die Röhren des Liebig'schen Trockenapparats verstopfte. Bei einigen wiederholten Darstellungen wurde nun das Asparagin in einer Abdampfschale im Wasserbad mit überschüssiger concentrirter Salzsäure gekocht, die möglichst trockene Masse in eine Retorte gebracht, durch deren Tubulus fortwährend ein Strom getrockneter Kohlensäure eingeleitet, und die Masse im Paraffinbade auf $120^{\circ} \mathrm{C}$. erhitzt. Nach etwa 10 stündigem Erhitzen wurde die Temperatur crhöht, worauf bei $140^{\circ}$ von Neuem Salzsäure und Wasser austrat, 
bis schliefslich, nach mehrtägigem Erhitzen und bis auf $200^{\circ}$ C. gesteigerter Wärme, die Masse trocken wurde und keine sauren Dämpfe mehr bemerklich waren.

Die fernere Behandlung war dieselhe wie oben. $30 \mathrm{Grm}$. gaben so etwa $34 \mathrm{Grm}$. der rohen Masse.

Es zeigte sich, dafs bei langsam steigender Temperatur und möglichst lange dauerndem Erhitzen (bis zu 6 Tagen) hauptsächlich die unlösliche Substanz gebildet wurde. Wird zu viel Asparagin in Arbeit genommen, oder gleich Anfangs zu rasch auf eine höhere Temperatur erwärmt, so bräunt sich dic Masse.

Analysen der in Wasser schwer löslichen Substanz.

$1,205 \mathrm{Grm}$. lufttrockener Substanz verloren bei $130^{\circ} \mathrm{C}$. getrocknet 0,116 Wasser, also $9,63 \mathrm{pC}$.

Ferner gaben 0,2842 Grm. Subslanz bei der Verbrennung mit Kupferoxyd, chlorsaurem Kali und vorgelegten Kupferspänen 0,493 Kohlensäure und 0,0945 Wasser.

Ferner gaben 0,3293 Grm. Substanz mit chromsaurem Blei, Kupferoxyd und vorgeleglen Kupferspänen verbrannt 0,5715 Kohlensäure und 0,112 Wasscr.

$0,3463 \mathrm{Grm}$. Substanz lieferten mit Natronkalk verbrannt 0,7698 Platinsalmiak und 0,3355 Platin, entsprechend 13,96 und 13,905 pC. Stickstoff.

Dieser Verbindung kommt folgende Formel zu: $\mathrm{C}_{16} \mathrm{H}_{14} \mathrm{~N}_{4} \mathrm{O}_{9}$; sie ist hiermit als ein Anhydrid anzusehen, welches 4 Mol. Asparaginsãure unter Wasseraustritt vereinigt enthält ; nämlich

$$
\begin{aligned}
& \mathrm{C}_{16} \mathrm{H}_{28} \mathrm{~N}_{4} \mathrm{O}_{16} \quad 4 \mathrm{Mol} \text {. Asparaginsüure } \\
& \mathrm{C}_{16} \mathrm{H}_{14} \mathrm{~N}_{4} \mathrm{O}_{8} \\
& \mathrm{II}_{14} \mathrm{O}_{7}
\end{aligned}
$$

treten als Wasser aus. 
Die procentische Berechnung ist folgende :

\begin{tabular}{|c|c|c|c|}
\hline \multicolumn{2}{|c|}{ Berechnet } & \multicolumn{2}{|c|}{ Gefunden } \\
\hline C & 47,30 & 47,30 & 47,33 \\
\hline $\mathrm{H}$ & 3,45 & 3,69 & 3,78 \\
\hline $\mathbf{N}$ & 13,80 & 13,96 & 13,90 \\
\hline o & 35,45 & - & - \\
\hline
\end{tabular}

Ein Theil der in Wasser schwerlöslichen Substanz wurde in Wasser und Ammoniak gelöst, das freie Ammoniak verdampft und nun mit Silberlösung versetzt. Von dem reichlich erfolgten gewaschenen und getrockneten Niederschlag gaben $0,2725 \mathrm{Grm}$. verbrannt 0,0485 Grm., entsprechend 17,8 pC. Silber.

Das Silbersalz enthält hiernach auf obige Menge mit $16 \mathrm{C}$ etwa 1 Aeq. Silber.

Die Analysen der in Wasser anlöslichen Substanzen ergaben Folgendes :

1,4025 Grm. der luftrockenen Substanz wogen nach dem Trocknen bei 100 bis $120^{\circ}$ C. 1,2295 Grm.; also Wasserverlust $12,3 \mathrm{pC}$.

Bei einer zweiten Portion von $0,4655 \mathrm{Grm}$. hinterblieben nach dem Trocknen 0,4345 Grm.; also Wasserverlust $12,5 \mathrm{pC}$.

Zur Ermittelung des Kohlenstoff- und Wasserstoffgehalts wurden 0,3315 Grm. des getrockneten Salzes mit Kupferoxyd (chlorsaurem Kali und vorgelegten Kupferspänen) verbrannt; diese gaben 0,5873 Grm. Kohlensăure und 0,1098 Grm. Wasser, oder 48,32 pC. Kohlenstoff und $3,68 \mathrm{pC}$. Wasserstoff.

Eine zweite Probe von 0,3273 Grm. Substanz gab mil chromsaurem Blei, Kupferoxyd und vorgelegten Kupferspānen verbrannt 0,5875 Grm. Kohlensäure und 0,1065 Grm. Wasser, also Kohlenstoff $48,95 \mathrm{pC}$, Wasserstoff $3,6 \mathrm{pC}$.

Bei der Stickstoffbestimmung mit Natronkalk gaben 0,2598 Grm. Substanz 0,5688 Grm. Plalinsalmiak oder 0,2495 
Grm. Platin; diefs ergiebt für Platinsalmiak 13,75 pC., für Platin 13,64 pC. Stickstoff.

Die Resultate dieser Analysen sprechen für folgende Formel :

$$
\begin{aligned}
& \mathrm{C}_{82} \mathrm{H}_{26} \mathrm{~N}_{8} \mathrm{O}_{17} \text {. } \\
& 8 \text { Mol. Asparaginsäure }=\mathrm{C}_{32} \mathrm{I}_{56} \mathrm{~N}_{8} \mathrm{O}_{92} \\
& \text { es traten also aus } \mathrm{H}_{30} \mathrm{O}_{15} \text {. }
\end{aligned}
$$

Die procentische Zusammensetzung dieses bei 100 bis $120^{\circ} \mathrm{C}$. getrockneten Producles ist also :

\begin{tabular}{cr} 
Berechnet \\
\cline { 1 - 1 } C & 48,36 \\
H & 3,30 \\
N & 14,10 \\
O & 34,24 \\
\hline & $-100,00$.
\end{tabular}

$$
\begin{aligned}
& \frac{\text { Gefunden }}{48,32} \frac{18,95}{48,08} \\
& 3,68 \quad 3,60 \\
& 13,75 \quad 13,64 \\
& \text { - }
\end{aligned}
$$

Der Wassergehalt der luftrockenen Substanz beträgt nach obiger Formel $12 \mathrm{Mol}$.

Die unlōsliche sowohl als auch die schwerlösliche Substanz sind daher aus Asparaginsäure unter Austreten von Wasser entstanden; sie gehen bei der Behandlung mit Ammoniak oder Barytwasser wieder in Asparaginsäure über. In Ammoniak lósen sie sich sehr leicht auf; die Lösung giebt mit Barytlösung einen schwerlöslichen Niederschlag, dessen Analyse zu keiner cinfachen Formel führt. Kocht man die ammoniakalische Lösung anhaltend mit Barytwasser und fällt hierauf den Baryt mit Schwefelsäure genau aus, so entstehen beim Verdunsten Krystalle von Asparaginsäure. Dieselben waren wasserfrei und zeigten folgende Zusammensetzung.

Bei der Verbrennung mil chromsaurem Bleioxyd, Kupferoxyd und vorgelegten Kupferspänen gaben $0,3812 \mathrm{Grm}$. der trockenen Substanz 0,5128 Grm. Kohlensäure und 0,1813 Grm. 
Wasser, entsprechend $36,69 \mathrm{pC}$. Kohlenstoff und $5,28 \mathrm{pC}$. Wassersioff.

Bei der Stickstoffbestimmung mit Natronkalk gaben $0,1604 \mathrm{Grm}$. Substanz 0,118 Grm. Platin, entsprechend 10,44 pC. Stickstoff.

Die hieraus berechnete Formel ist $\mathrm{C}_{4} \mathrm{H}_{7} \mathrm{NO}_{4}$, also die der Asparaginsäure.

\begin{tabular}{lrc}
\multicolumn{2}{c}{ Berechnet } & Gefunden \\
$\mathrm{C}$ & 36,1 & 36,69 \\
$\mathrm{H}$ & 5,3 & 5,28 \\
$\mathrm{~N}$ & 10,6 & 10,44 \\
$\mathrm{O}$ & 48,0 & - \\
\cline { 2 - 3 } & $100,0$. &
\end{tabular}

Kupfersalz aus Asparaginsäure.

Sowohl beim Kochen der Mutterlauge als auch dieser weifsen Krystalle mit gefälltem Kupferoxyd wurde ein schwer lösliches, in hübschen Nadeln krystallisirendes ultramarinblaues Salz erhalten. Dieses Salz verlor bei 100 bis $110^{\circ} \mathrm{C}$. $21,09 \mathrm{pC}$. Wasser. Der Kupfergehalt in dem so getrockneten Salz betrug $29,94 \mathrm{pC}$; bei 120 bis $130^{\circ} \mathrm{C}$. längere Zeit getrocknet verlor es $32,53 \mathrm{pC}$. Wasser, zeigte aber schon leichte Spuren von Zersetzung; bis $120^{\circ} \mathrm{C}$. hatte es $27 \mathrm{pC}$. Wasser verloren.

Die Angaben von Dessaignes, dafs das asparaginsaure Kupfer bei $160^{\circ} \mathrm{C}$. getrocknet werden könne, haben sich bei mir nicht bewährt, indem drei Proben zwischen 130 und $140^{\circ} \mathrm{C}$. sich zersetzten und bei einer vierten bei $128^{\circ} \mathrm{C}$. die Zersetzung begaun.

Der Kupfergehalt des bei 120 bis $130^{\circ} \mathrm{C}$. getrockneten Salzes betrug $34,32 \mathrm{pC}$. Hiermit stimmt das schon bekannte asparaginsaure Kupferoxyd annähernd. Es enthält nämlich $5 \mathrm{Mol} .=31,64$ pC. Krystallwasser, und 32,4 pC. Kupfer im 
wasserfreien Salze. Der etwas gröfsere Verlust beim Trocknen, sowie in Folge davon der gröfsere Kupfergehalt obiger Analyse erklären sich aus der beginnenden Zersetzung des Salzes.

Weder das Zinksalz noch das Cadmiumsalz dieser Asparaginsäure konnte in Krystallen erhalten werden.

Das neutrale Silbersalz wurde erhalten durch Fällen der schwach alkalisch rengirenden Lösung von Asparaginsäure in verdünntem Ammoniak mit salpetersaurem Silberoxyd. Ein Ueberschufs von Ammoniak oder Silberlösung löst den Niederschlag wieder auf. Das weifse körnige Salz wurde über Schwefelsäure getrocknet.

$4,7175 \mathrm{Grm}$. verloren im Lufibade bei 90 bis $100^{\circ} \mathrm{C}$. getrocknet $0,017 \mathrm{Grm}$. Wasser, also $0,36 \mathrm{pC}$.

$0,6598 \mathrm{Grm}$. des Silbersalzes gaben geglüht 0,4102 Grm., also $62,17 \mathrm{pC}$. Silber.

Nach der Formel $\mathrm{C}_{4} \mathrm{H}_{5} \mathrm{Ag}_{2} \mathrm{NO}_{4}$ heträgt der Silbergehalt $62,25 \mathrm{pC}$.

Das optische Verhalten dieser Asparaginsäure.

Die Lösung dieser Asparaginsäure in Ammoniak erwies sich als nicht circularpolarisirend.

Eben so verhielt sich die ammoniakalische Lösung der in Wasser unlōslichen Substanz.

Die aus Asparaginsäure unter Austreten von Wasser entstehenden Anhydride nähern sich in der Zusammensetzung den einfachen, durch Austreten von 1 oder 2 Al. Wasser aus einem Molecul Asparaginsäure abzuleitenden Anhydriden $\mathrm{C}_{4} \mathrm{H}_{5} \mathrm{NO}_{3}$ und $\mathrm{C}_{4} \mathrm{H}_{3} \mathrm{NO}_{2}$, welche in 100 Theilen verlangen :

\begin{tabular}{|c|c|c|c|}
\hline$C_{4}$ & 41,70 & $C_{4}$ & 49,50 \\
\hline $\mathrm{H}_{5}$ & 4,35 & $\mathrm{H}_{3}$ & 3,10 \\
\hline $\mathrm{N}$ & 12,18 & $N$ & 14,40 \\
\hline $\mathrm{O}_{\mathbf{s}}$ & 41,70 & $\mathrm{O}_{2}$ & 33,00 \\
\hline & $00, \overline{0} 0$ & & 100,00 \\
\hline
\end{tabular}


Sie sind übrigens unzweifelhaft Condensationsproducte und enthalten stets $\mathrm{n}_{4}$ auf $\mathrm{n} \mathrm{N}$. Sie gehen daher auch bei der Behandlung mit Ammoniak unter vorübergehender Bildung von complicirteren Producten, deren Baryumverbindungen in Wasser theils unlöslich, theils schwerlöslich sind, zuletzt in Asparaginsäure über.

Achnliche Körper wurden von Dessaignes*) durch Erhitzen von äpfelsaurem Ammoniak auf $200^{\circ} \mathrm{C}$. erhalten; eben so erhielt Paste ur ${ }^{* *}$ ) eine in Wasser unlösliche Substanz, deren Zusammenselzung der Formel $\mathrm{C}_{8} \mathrm{H}_{8} \mathrm{~N}_{z} \mathrm{O}_{5}$ entsprach; J. Wolff endlich bekam nach einem ähnlichen Verfahren aus äpfelsaurem Ammoniak eine in Wasser schwerlösliche Substanz, deren Zusammensetzung sich der Formel $\mathrm{C}_{4} \mathrm{H}_{3} \mathrm{NO}_{2}$ nähert. Diese letzteren Bildungsweisen gehören zu den interessanteren, weil selteneren Unsetzungen, bei welchen ein Austausch der. Atomgruppen im Molecul stattindet. Sie sind in dieser Hinsicht durchaus analog der Entstehung des Taurins durch Erhitzen von isäthionsaurem Ammoniak. Der Stickstoff, welcher zunächst als Ammonium mit Sauerstoff und Carboxyl verbunden war, trat in directe Verbindung mit dem Kohlenstoff des Kerns über. Es ergiebt sich diefs daraus, dafs diese Producte durch Aufnahme von Wasser nicht in Aepfelsäure und Ammoniak, sondern in Asparaginsäure übergehen.

Aepfelsaures Anmoniak

$$
\mathrm{C}_{2} \mathrm{H}_{3}\left\{\begin{array}{l}
\mathrm{CO} . \mathrm{OH} \\
\mathrm{OH} \\
\mathrm{CO} . \mathrm{ONH}_{4}
\end{array}\right.
$$

giebt zuletzt unter Austreten von Wasser

$$
\mathrm{C}_{2} \mathrm{H}_{2}\left\{\begin{array}{l}
\mathrm{CO} \\
\mathrm{NH} \\
\mathrm{CO}
\end{array}\right\} \text {. }
$$

\footnotetext{
*) Jahresbericht für Chemie u. s. w. f. 1850, 414.

**) Daselbst f. 1857, 309 .

***) Daselbst f. 1850,415 .
} 
Diese Verwandlung erfolgt aber nur Jurch Zusammentreten mehrerer Molecule unter Bildung condensirterer Producte.

Die vorstehende Arbeit wurde im Laboratorium des Herrn Prof. Dr. Strecker ausgeführt. Für seine freundlicho Unterstützung sage ich demselben meinen besten Dank.

\section{Ueber Brenzweinsulfosäure;}

von Dr. Theodor Wieland.

Verschiedene bereits vorliegende Thatsachen *) scheinen darauf hinzudeuten, dafs die ungesättigten Kohlenstoffrerbindungen, in welchen naan zwei Atome Kohlenstoff doppelt gebunden annehmen kann, ähnlich wie sie sich direct mit 2 At. Wasserstoff, Chlor oder Brom, oder auch mit 1 Mol. Chlor-, Brom- oder Jodwasserstofl vercinigen, so auch 1 Mol. saures schwefligsaures Alkali direct aufnehmen können. Zu diesen Körpern gehören auch die drei isomeren Brenzcitronensüuren, $\mathrm{C}_{5} \mathrm{H}_{6} \mathrm{O}_{4}$, und ich habe daher auf die Aufforderung und unter Leitung des Herrn Prof. Strecker das Verhalten dieser Säuren gegen schwefligsaure Alkalien näher untersucht. Ich befolgte hierbei folgendes einfache Verfahren. Eine Lösung von neutralem schwefligsaurem Kali $\left(\mathrm{KSO}_{3} \mathrm{~K}\right)$ wurde mil 1 Mol. der betreffenden Säure in mäfsig concentrirter Lösung in einer Retorte mit aufwärts gerichtetem Hals und damit verbundenem Rückflufskühler einige Stunden lang

*) Vgl. Valet, iiber Phenylpropionsäurc; diese Annalen CLIV, 62; Credner, über Sulfofumarsïure, Inauguraldissertation, Tübingen 1869. 